\title{
ORIGINAL ARTICLE \\ Rectal hyposensitivity for non-noxious stimuli, postprandial hypersensitivity and its correlation with symptoms in complete spinal cord injury with neurogenic bowel dysfunction
}

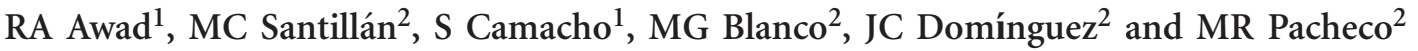

\begin{abstract}
Study design: Prospective clinical study.
Objectives: To assess fasting and postprandial (PP) perception of rectal distension and its correlation with symptoms in patients with spinal cord injury (SCl) and neurogenic bowel dysfunction compared to ten healthy subjects (HS).

Setting: Experimental Medicine and Motility Unit, Mexico General Hospital and National Institute of Rehabilitation.

Methods: Twenty patients with complete SCI at cervical, thoracic and lumbar levels [American Spinal Injury Association (ASIA) A] were studied. Rectal sensitivity was evaluated with a barostat.

Results: In SCl patients, while lower the rectal tone more time was used for defecate $(R=0.50, P=0.048)$ and more PP episodes of fecal incontinence occur $(R=0.54, P=0.030)$. The thresholds for non-noxious stimuli of first $(23.6 \mathrm{mmHg}, \mathrm{Cl} 19.5-27.7)$ vs 14.0 (Cl 10.9-17.1), $P=0.004$; gas $(27.9 \mathrm{mmHg}, \mathrm{Cl} 19.9-35.8)$ vs $17.9 \mathrm{mmHg}(\mathrm{Cl} 14.25-21.69), P=0.02$ and urge-to-defecate sensation (33.2 mmHg, $\mathrm{Cl} 27.5-38.8)$ vs $22.4 \mathrm{mmHg}(\mathrm{Cl} 17.9-26.9), P=0.01$ were reported by SCl patients at higher pressure than $\mathrm{HS}$, respectively. $\mathrm{SCl}$ patients reported PP pain sensation at a lower pressure than controls $(27.8 \mathrm{mmHg}, \mathrm{Cl} 21.5-34.2$ vs $36.5 \mathrm{mmHg}, \mathrm{Cl} 31.8-41.2), P=0.04$.

Conclusion: $\mathrm{SCl}$ patients preserve rectal sensation, present rectal hyposensitivity for non-noxious stimuli and PP hypersensitivity. Lower rectal tone was related to the time used for defecate and with fecal incontinence. The results suggest that an intact neural transmission between the spinal cord and higher centres is indispensable for noxious stimulus, but not for non-noxious stimuli. Also, barostat sensitivity studies can complement ASIA criteria to verify a complete injury.
\end{abstract}

Spinal Cord (2013) 51, 94-98; doi:10.1038/sc.2012.98; published online 28 August 2012

Keywords: visceral sensitivity; barostat; neurogenic bowel dysfunction; spinal cord injury

\section{INTRODUCTION}

Traumatic spinal cord injury (SCI) represents a significant public health problem worldwide. ${ }^{1}$ About half of those with SCI exhibited neurogenic bowel dysfunction $(\mathrm{NBD})^{2}$ which affects the entire gastrointestinal (GI) tract. Moreover, SCI subjects may develop autonomic dysreflexia in response to noxious stimulus. Cardiovascular dysregulation, characterized by paroxysmal high blood pressure episodes, is the most prominent feature and is precipitated by manual emptying of rectal contents and by gastric and bowel distension. ${ }^{3}$ Studies have shown a range of physiological affects such as abnormal oesophageal contraction, ${ }^{4}$ reduction in gastric emptying, ${ }^{5}$ decrease of phase III of the interdigestive motor complex, prolonged orocecal transit time, ${ }^{6}$ suboptimal postprandial (PP) colonic response ${ }^{7}$ and spino-anorectal neuropathy. ${ }^{8}$ However, physiologic studies regarding visceral sensation are lacking. In patients with SCI, it is important to determine the integrity of the afferent and efferent neurotransmission involved in daily physiological events. It is possible to assess the response to food, and presence of rectal non-noxious sensation (first sensation, gas sensation and need to defecate sensation) and the noxious sensation of pain. The combined approach, assessing rectal sensitivity during both fasting and PP periods, provides very useful information about the pathophysiology of constipation, which is frequent in SCI patients. We evaluated rectal tone after food, which in healthy subjects showed no variation but which in constipated patients increased significantly as measured by the baseline bag volume, indicating that food diminished rectal tone in constipated patients. ${ }^{9}$ Suttor et al. ${ }^{10}$ reported that intact neural transmission between the spinal cord and higher centres are not essential for normal colorectal motor response. On the other hand, visceral sensitivity testing may be a future requirement, in addition to the American Spinal Injury Association (ASIA) criteria, to assess the completeness of cord lesions in patients diagnosed with complete spinal cord transsection, as some subjects report the sensation of distension of the rectum. ${ }^{11}$ In the current study, our outcome measures assessed fasting and PP perception of rectal distension in 20 patients with complete supraconal SCI (lesions

${ }^{1}$ Experimental Medicine and Motility Unit, Gastroenterology Service U-107, Mexico City General Hospital, México, DF, México and ${ }^{2}$ Spinal Cord Injury Section, National Institute of Rehabilitation, Mexico, Mexico

Correspondence: Professor RA Awad, Experimental Medicine and Motility Unit, Gastroenterology Service U-107, Mexico City General Hospital, Dr Balmis \#148, Col. Doctores, 06726 México, DF, México.

E-mail: awadrichardalexander@prodigy.net.mx

Received 13 March 2012; revised 2 August 2012; accepted 3 August 2012; published online 28 August 2012 
above the conus medullaris, which left the sacral outflow from the spinal cord intact ${ }^{12}$ ) at different levels, and with NBD, compared to ten healthy subjects (HS).

\section{MATERIALS AND METHODS}

Subjects

Patients were recruited from the SCI Section at National Institute of Rehabilitation, Mexico. The study was conducted at the Experimental Medicine and Motility Unit in the Gastroenterology Service of Mexico City General Hospital. The Ethical and Research Committee of Mexico City General Hospital approved the protocol, and signed informed consent was obtained from all subjects and treating physicians. The data of all enlisted consecutive outpatients presenting with complete SCI during a nine-month period at our motility-based problems tertiary unit were entered into a database for further analysis. Inclusion criteria were age over 18 years, NBD (bowel dysfunction score: 0-6 very minor, 7-9 minor, 10-13 moderate, and 14 or more severe. The cut off score used was moderate), ${ }^{13}$ and fulfilment of the ASIA criteria for complete supraconal SCI for a minimum of 12 months. The neurology level was determined by members of the SCI section at Mexico National Institute of Rehabilitation (MCS, MGB, JCD, MRP) according to the ASIA Impairment Scale (AIS) and the International Standards for Neurological Classification of SCI criteria for complete SCI (AIS: A $=$ Complete: No motor or sensory function is preserved in the sacral segments S4-S5). Exclusion criteria included previous anorectal surgery, concomitant treatments affecting smooth muscle during the past month, pregnant or nursing, any concomitant disease, other neurological lesions, significant autonomic dysreflexia, major pressure ulceration, marked psychiatric alterations (anxiety disorders, major depression, or formal psychiatric illness), patients who had participated in a clinical trial over the past two months, and clinically relevant deviations from the normal range of laboratory results. Patients were included after a 15-day period during which the usual bowel management of the patients was recorded daily with a diary. Ten HS formed the control group. All patients underwent evaluation of clinical history, blood count with chemistry panel, three coproparasitoscopic studies, wet mount preparation for amoeba, rectosigmoidoscopy (Welch Allyn 32823 sigmoidoscope, Skaneateles Falls, NY, USA), and barostat rectal sensitivity studies.

\section{Study protocol}

Visceral afferent sensation was assessed using an electronic barostat-distender assembly (Distender II; G. \& J. Electronics, Toronto, Canada) in the rectum. ${ }^{14}$ The barostat is a device that maintains a constant pressure in an air-filled bag through a feedback mechanism. The barostat bag consisted of a cylindrical ultra-thin polyethylene bag of infinite compliance having a maximum inflation of $600 \mathrm{ml}$, which was attached at both ends to a single-lumen polyvinyl catheter connected to a $16 \mathrm{Fr}$ flexible double-lumen polyvinyl catheter (Mui Scientific, Ontario, Canada). The bag was checked for leaks at the beginning and after completion of each test. Data were downloaded and stored onto a computer for later analysis. Subjects were administered a tap-water enema 60 120 min prior to insertion of the barostat bag into the rectum. The barostat was allowed to warm up for $1 \mathrm{~h}$ before the study. All patients were studied after an overnight fast, in a quiet, isolated room, without sedation in the left lateral decubitus position with distal end of the bag positioned $5 \mathrm{~cm}$ from the anal verge. Patients were instructed concerning the nature of the distension protocols, and had no visual or auditory cues to anticipate the intensity of distension. The individual operating pressure (IOP), that is, the minimum pressure required to overcome passive resistance to bag inflation ${ }^{15}$ was determined by inflation of the bag in a stepwise fashion, beginning with $6 \mathrm{mmHg}$ and increasing by $2 \mathrm{mmHg}$ every $30 \mathrm{~s}$. The procedure continued until the volume and pressure tracings began to show respiratory variation. After obtaining the IOP, the bag was inflated two steps up to ensure that the bag was totally unfolded and in complete contact with the subject's rectum. To test muscle tone, the bag was inflated to the IOP and the volume was recorded over a $15 \mathrm{~min}$ period.

\section{Distension protocol}

Sensory thresholds were determined using the ascending method of limits protocol, as reported previously by the current authors and many others researchers. ${ }^{9,15-19}$ The barostat was programmed to deliver a series of phasic isobaric rectal distensions $60 \mathrm{~s}$ in duration, separated by $60 \mathrm{~s}$ periods, during which bag pressure returned to the IOP. Distension stimuli were programmed to increase by $4 \mathrm{mmHg}$ with each distension until the subject reported pain. Sensation in response to distension was determined by means of a patient Perception Panel (G. \& J. Electronics) interfaced with the software of the barostat. The questionnaire for sensation consisted of a graphic rating scale with combined numerical descriptors on a visual analogue scale graded independently from one to seven (1 represented first/initial sensation, 2 represented gas sensation, 3 represented urgency, 4 represented the first sensation of pain and 5 to 7 represented successive grades of pain). Each trial was complete when either the patient pressed the panic button or a pressure of $48 \mathrm{mmHg}$ was achieved; ${ }^{17}$ usually, the bag was deflated when patients reported the first sensation of pain. It was emphasized to the subject that they had full control over the protocol through their ability to deflate the bag instantaneously at any time of significant discomfort or pain. The procedure was repeated $30 \mathrm{~min}$ after ingestion of standardized food previously shown by means of electrical and mechanical registers to elicit gastrocolonic response to food. ${ }^{20}$

\section{Statistical analysis}

Outcome measures were the assessment of fasting and PP perception of rectal distension in 20 patients with complete SCI and NBD compared with ten HS. A binominal 95\% confidence interval was calculated for all variables. Data are presented as mean ( $95 \% \mathrm{CI})$. An alpha level of 0.05 and paired and non-paired Student two-tailed $t$-tests were used. Associations between symptoms and rectal tone and sensitivity were done with Spearman's correlation. Statistical analyses were calculated using the 2000 GraphPad Software package of statistical programs (San Diego, CA, USA).

\section{RESULTS}

\section{Subjects}

Twenty patients (36 \pm 11 , range 23-63 years, 16 male and four female) were enrolled into the study, and were compared with ten asymptomatic HS ( $25 \pm 7$, range 18-41 years, seven males, three female). Body mass index was similar in the SCI patients compared with HS, $24 \pm 4.5$ vs $26 \pm 5$ weight in $\mathrm{kg}$ per height in $\mathrm{m}^{2}$, respectively. Time since injury was over two years [ $4.7 \pm 3.2$ (CI 3.2-6.3) years]. The level of lesion was thoracic in $11(11 / 20,55 \%)$, cervical in $7(7 / 20,35 \%)$ and lumbar in $2(2 / 20,10 \%)$. Lesions were all caused by trauma and were complete in all patients (American Spinal Injury Association impairment scale A). The most common intervention was digital stimulation of the anorectum once or more every week with 31 to $60 \mathrm{~min}$ used for each defecation. None of the patients perceived the normal desire of defecation. Textually they described the feeling of defecation as abdominal distension, hard and cool abdomen, hardening of the legs, abdominal pain, chills and dizziness, itching in the head, and feeling of pain at the sacrum level. All patients suffered from a combination of constipation and fecal incontinence, and the NBD score was $15 \pm 5$; CI: 12.9-17.7 (Krogh et al. ${ }^{13}$ ). None of all the studied clinical and physiological variables changed in connection with the duration of SCI or age of the patients. The laboratory test parameters and recto-sigmoidoscopy carried out on all patients were normal.

\section{Rectal tone response to rectal distension and meal ingestion} IOP was higher in SCI patients $(12 \pm 2 \mathrm{mmHg}$, CI: 11-13) compared with HS $(9.5 \pm 2 \mathrm{mmHg}$, CI: $8-10 ; P=0.003)$. Fasting and PP rectal mean bag volume (that is, smooth muscle tone) and rectal compliance ( $\mathrm{ml}$ per $\mathrm{mmHg}$ ) were similar in both SCI patients and HS (Table 1). 


\begin{tabular}{|c|c|c|c|c|}
\hline & \multicolumn{2}{|c|}{ Fasting } & \multicolumn{2}{|c|}{ Posprandial } \\
\hline & $S C l(n=20)$ & Healthy subjects $(\mathrm{n}=10)$ & $\mathrm{SCl}$ & Healthy subjects \\
\hline Tone $(\mathrm{ml})$ mean $(95 \% \mathrm{Cl})$ & $115.9(76.2-155.6)$ & $103.5(71.8-135.1)$ & $142.7(103.4-182)$ & $108.1(71.4-144.8)$ \\
\hline Compliance $(v / p)$ mean $(95 \% \mathrm{Cl})$ & $9.5(6.2-12.7)$ & $11.8(7.5-16.2)$ & $11.7(8.4-14.9)$ & $12.1(7.4-16.8)$ \\
\hline First sensation $(\mathrm{mmHg})$ mean $(95 \% \mathrm{Cl})$ & $23.6(19.5-27.7)^{*}$ & $14(10.9-17.1)$ & $23(19.6-26)^{*}$ & $13.4(11-15.9)$ \\
\hline Gas sensation $(\mathrm{mmHg})$ mean $(95 \% \mathrm{Cl})$ & $27.9(19.9-35.8)^{*}$ & $17.9(14.2-21.6)$ & $23.8(8.3-39.2)$ & $18.77(15.8-21.7)$ \\
\hline Urge defecate $(\mathrm{mmHg})$ mean $(95 \% \mathrm{Cl})$ & $33.2(27.5-38.8)^{*}$ & $22.4(17.9-26.9)$ & $24.7(19.6-29.8)$ & $27.8(23.5-32.1)$ \\
\hline Pain sensation $(\mathrm{mmHg})$ mean $(95 \% \mathrm{Cl})$ & $35.2(27.2-43.13)$ & $35.9(30.4-41.3)$ & $27.8(21.5-34.2)^{*}$ & $36.58(31.8-41.2)$ \\
\hline
\end{tabular}

Comparison with healthy subjects: ${ }^{*} P<0.05$.

Nevertheless, in SCI patient's rectal tone decreased $(P=0.023)$ after food compared with their own fasting values (Table 1).

\section{Sensory thresholds to rectal distension and meal ingestion}

Table 1 show that fasting and PP sensory thresholds during phasic, isobaric distension were significantly different between groups. The SCI patients had higher first sensation thresholds compared with the HS in both fasting $(P=0.004)$ and satiated $(P=0.0008)$ states. In SCI patients compared with HS the thresholds for non-noxious stimuli were higher in fasting gas sensation $(P=0.024)$ and in fasting urgeto-defecate sensation $(P=0.010)$. Fasting pain sensation was similar in both groups; however, SCI patients reported PP pain sensation at lower pressure than controls $(P=0.043)$. One of the seven cervical SCI patients reported pain sensation. Compared with their own fasting values there was no significant change in the PP threshold for noxious and non-noxious stimuli in both groups. Except for the PP urge to defecate sensation (Table 1), which was felt at a lower pressure for the SCI patients $(P=0.012)$ and at a higher pressure for the HS $(P=0.002)$.

\section{Correlation between symptoms with rectal tone and sensitivity} Some symptoms correlated statistically with rectal tone. Fasting rectal bag volume (high bag volume indicates diminished smooth muscle tone in the rectum) was positively correlated with the time used for each defecation $(r=0.5009, P=0.048)$, and negatively correlated with flatus incontinence $(r=-0.5429, P=0.036)$. PP rectal bag volume was positively correlated with the frequency of fecal incontinence $(r=0.54, P=0.030)$ and negatively correlated with flatus incontinence $(r=-0.587, P=0.021)$. None of the symptoms was correlated with any of the sensitivity parameters.

\section{DISCUSSION}

The interesting findings of this study are that SCI patients preserve rectal sensation, present rectal hyposensitivity for non-noxious stimuli, postprandial hypersensitivity, and symptoms correlated statistically with rectal tone. In the current study SCI patients showed no differences in rectal tone compared with HS. These data disagree in part with Krogh et al. ${ }^{12}$ who reports that rectal tone is higher in patients with supraconal lesions but lower in conal/cauda equina lesions. Differences in methodology and selection of patients may account for this discrepancy, as we utilized a barostat technique to analyse 20 patients with complete SCI, and they used rectal impedance planimetry in only five patients. ${ }^{12}$ On the other hand, in SCI patients's rectal tone decreased after food compared with their own fasting values. The presence of impaired gastrorectal response in our patients disagrees in part with a recent report ${ }^{10}$ in which four out of six cervical SCI patients and five HS showed an increase in rectal tone, however, with no group effect in the second $30 \mathrm{~min}$. As for HS, this data supports previous results showing that rectal tone does not vary after food. ${ }^{9,16}$ Interestingly, in our study some symptoms and incontinence parameters correlated statistically with rectal tone. In SCI patients while lower the rectal tone more time was used for each defecation. This could be related to a lesser or difficult contact with the receptors at the rectal wall, which strengthen the participation of the rectum in the mechanism of defecation and agree with the concept that anorectal filling sensations and continence require a rectal reservoir of adequate capacity. ${ }^{21}$ After food, increased episodes of solid fecal incontinence were related to lower rectal tone. The clinical implications of these findings are that SCI patients may present more episodes of fecal incontinence after food. The pathophysiology of fecal incontinence is not well known. Recently it is reported that fecal incontinence is associated with increased rectal stiffness, ${ }^{22}$ however, our rectal tone data do not confirm that assumption. Besides, Peirce et al. ${ }^{23}$ in an experimental model of neuropathic fecal incontinence quantifying the effect of crush and compression injury on first-order sensory neurons of the inferior rectal nerve, confirmed the presence of axonal injury, principally of the C-fibre afferent pathways. The new data in our unfortunate SCI human model may contribute to the understanding of the pathophysiology of fecal incontinence.

Sensory thresholds during phasic, isobaric distension were significantly different between SCI and HS. For fasting, thresholds for nonnoxious stimuli of first, gas, and urge-to-defecate sensation were higher in SCI patients compared with HS. In this regard, as established by Gladman et al. ${ }^{24}$ rectal hyposensitivity relates to a diminished perception of rectal distension. Rectal hyposensitivity is reported in some patients with constipation, fecal incontinence ${ }^{25}$ and with obstructed defecation. ${ }^{26}$ This agrees in part with the present findings as our patients present with constipation and fecal incontinence and suggests that rectal hyposensitivity is a characteristic feature of patients with SCI. On the other hand, as in our SCI patients rectal compliance was not different than values obtained in HS, rectal hyposensitivity may reflect an impaired afferent nerve pathway rather than presence of abnormal rectal wall properties. ${ }^{24,26}$ In addition, these data suggest that rectal fasting sensory thresholds for nonnoxious stimuli are inhibited by supraspinal centers within the CNS. In concordance with this, Krogh et al. ${ }^{12}$ reported that rectal contractility is inhibited by supraspinal centers. On the other hand, the noxious stimulus of pain sensation during the fasting state exhibited no difference between SCI and controls. This supports the concept that noxious and non-noxious distension stimulates different afferent nerve pathways. On the contrary, during PP state, in SCI patients the threshold for pain sensation was lower than for HS, which suggests that SCI patients with supraconal lesions may present 
with PP visceral hypersensitivity. The increased sensitivity might be caused by loss of inhibitory input from supraconal centres in the CNS, which is the same explanation that Krogh et al. ${ }^{12}$ assumed for the increased frequency of giant rectal contractions in SCI patients. This demonstrates that abnormal rectal sensory processing, which is known to occur in patients suffering from constipation ${ }^{16}$ is also present in patients suffering from supraconal SCI.

Only one of the seven cervical SCI patients reported pain sensation, which implies that for noxious stimulus an intact neural transmission between the spinal cord and higher centres is essential. In contrast, Suttor et al. ${ }^{10}$ claim that an intact neural transmission between the spinal cord and higher centres is not essential for a normal colorectal motor response to feeding. The discrepancy may lie in that they elicit non-noxious stimulus.

We are aware ${ }^{9}$ that the power of this study is limited by the number of subjects and that a small study is particularly susceptible to a typeII error; however, it is reported that power is then appropriately indicated by confidence intervals, ${ }^{27}$ which was the case in the present study. In addition, our study analysed 20 subjects, while in 14 papers dealing with physiological aspects of $\mathrm{SCI}^{10,11}$ the median sample size was $13 \pm 9.7$ (CI 8.0-18.2) patients.

All of our patients classified as having a complete spinal cord transection according to ASIA reported sensation in response to noxious and non- noxious stimuli. This data suggest that presence of anorectal sensation may indicate incomplete SCI. Wietek et al. ${ }^{11}$ investigated 11 paraplegic patients during non-painful stimulation of the distal rectum and anal canal, and using event related functional magnetic resonance imaging observed that complete SCI patients demonstrated cortical activation in areas similar to those activated in HS. ${ }^{11}$ To ensure the completeness of lesion is crucial, as the hypothesis for neurotransmission may vary considerably depending on that. We believe that, indeed, the lesion is clinically complete, but by other newly adapted routes or local neural pathways part of the afferent and efferent message are driven and the enteric nervous system in a paracrine fashion performs the main activity as locally as possible by means of intrinsic excitatory and inhibitory pathways. This may be very interesting from a clinical point of view, since the phenomenon of constipation and diarrhoea may be orchestrated in situ. This idea supports in part the impression of other investigators in the sense that chronic constipation may be at least partly attributable to anorectal dysfunction after SCI. ${ }^{10}$

If so, the pharmacological approach in SCI patients must be redirected in order to act in a localized manner. Topical rectal therapies as first-line agents are at present recommended in patients with disease limited to the left colon or with active rectal inflammation. ${ }^{28}$ Methods of rectal administration include suppositories, liquid enemas, and foams. It is reported that rectal delivery modalities offer the advantage of delivering high local concentrations of active medication with minimization of systemic side effects. ${ }^{28}$ So, in light of our findings rectal delivery modalities should be consider more when prescribing drug therapy in patients with SCI.

Several hypotheses from other investigators support ours. Suttor et al. ${ }^{10}$ maintains that the preservation of the colorectal reflex and the colonic tonic response to a meal in cervical SCI indicates that these reflexes do not depend on neural transmission through central spinal pathway, rather they use intrinsic nerves or pre-vertebral pathways for colorectal reflex, and vagal and neurohormonal pathways for gastrocolonic reflex. In a PITH (destruction of the lumbar and sacral cords) guinea pig model it has been demonstrated that the intrinsic rectorectal and rectoanal inhibitory reflexes remain unchanged after removal of the extrinsic sacral excitatory neural pathway (pelvic nerves), ${ }^{29}$ suggesting that intrinsic cholinergic excitatory and nitrergic inhibitory pathways mediate the reflexes. ${ }^{29}$

In conclusion, our study provides very interesting findings. SCI patients preserve rectal sensation, present rectal hyposensitivity for non-noxious stimuli, PP hypersensitivity and symptoms correlated with rectal tone. The results from this study suggest that an intact neural transmission between the spinal cord and higher centres is essential for noxious stimulus, but not for non-noxious stimuli, and that incontinence and constipation may not be related solely to continuity of the spinal cord. If the enteric nervous system acts locally by means of intrinsic excitatory and inhibitory pathways, the pharmacological approach must be redirected to act in a localized delivery manner. Besides, barostat sensitivity studies can complement ASIA criteria to verify a complete injury.

\section{DATA ARCHIVING}

There were no data to deposit.

\section{CONFLICT OF INTEREST}

The authors declare no conflict of interest.

\section{ACKNOWLEDGEMENTS}

Ethics approval: The Ethical and Research Committee of Mexico City General Hospital approved the protocol, and signed informed consent was obtained from all subjects and treating physicians. Part of this work was presented at the 2009 and 2008 United European Gastroenterology Week and published in abstract form: GUT 2009;58:A327 and GUT 2008;57,Sup II:A77.

Author contributions: RAA was involved in creating the protocol, evaluation of data, recruitment of patients, acquisition of results, statistical analysis, and drafting of the manuscript. SC was involved in recruitment of patients, acquisition of results, and statistical analysis, while he was a Master of Sciences student. CS, MGB, JCD, and MRP were involved in recruitment of patients and acquisition of results. All authors have seen and approved the final version of the report.

1 DeVivo MJ. Epidemiology of traumatic spinal cord injury: trends and future implications. Spinal Cord 2012; 50: 365-372.

2 Awad RA. Neurogenic bowel dysfunction in patients with spinal cord injury, myelomeningocele, multiple sclerosis and Parkinson's disease. World J Gastroenterol 2011; 17: 5035-5048

3 Furusawa K, Tokuhiro A, Sugiyama H, Ikeda A, Tajima F, Genda E et al. Incidence of symptomatic autonomic dysreflexia varies according to the bowel and bladder management techniques in patients with spinal cord injury. Spinal Cord 2011; 49: 49-54.

4 Stinneford JG, Keshavarzian A, Nemchausky BA, Doria MI, Durkin M. Esophagitis and esophageal motor abnormalities in patients with chronic spinal cord injuries. Paraplegia 1993; 31: 384-392.

5 Qualls-Creekmore E, Tong M, Holmes GM. Time-course of recovery of gastric emptying and motility in rats with experimental spinal cord injury. Neurogastroenterol Motil 2010; 22: 62-68.

6 Fynne L, Worsoe J, Gregersen T, Schlageter V, Laurberg S, Krogh K. Gastric and small intestinal dysfunction in spinal cord injury patients. Acta Neurol Scand 2012; 125: 123-128.

7 Fajardo NR, Pasiliao RV, Modeste-Duncan R, Creasey G, Bauman WA, Korsten MA. Decreased colonic motility in persons with chronic spinal cord injury. Am J Gastroenterol 2003; 98: 128-134.

8 Tantiphlachiva K, Attaluri A, Valestin J, Yamada T, Rao SS. Translumbar and transsacral motor-evoked potentials: a novel test for spino-anorectal neuropathy in spinal cord injury. Am J Gastroenterol 2011; 106: 907-914.

9 Awad RA, Camacho S. A randomized, double-blind, placebo-controlled trial of polyethylene glycol effects on fasting and postprandial rectal sensitivity and symptoms in hypersensitive constipation-predominant irritable bowel syndrome. Colorectal Dis 2010; 12: 1131-1138.

10 Suttor VP, Ng C, Rutkowski S, Hansen RD, Kellow JE, Malcolm A. Colorectal responses to distension and feeding in patients with spinal cord injury. Am J Physiol Gastrointest Liver Physiol 2009; 296: G1344-G1349.

11 Wietek BM, Baron $\mathrm{CH}$, Erb M, Hinninghofen $\mathrm{H}$, Badtke A, Kaps HP et al. Cortical processing of residual ano-rectal sensation in patients with spinal cord injury: an fMRI study. Neurogastroenterol Motil 2008; 20: 488-497. 
12 Krogh K, Mosdal C, Gregersen H, Laurberg S. Rectal wall properties in patients with acute and chronic spinal cord lesions. Dis Colon Rectum 2002; 45: 641-649.

13 Krogh K, Christensen P, Sabroe S, Laurberg S. Neurogenic bowel dysfunction score. Spinal Cord 2006; 44: 625-631.

14 Whitehead WE, Delvaux M. Standardization of barostat procedures for testing smooth muscle tone and sensory thresholds in the gastrointestinal tract. The Working Team of Glaxo-Wellcome Research, UK. Dig Dis Sci 1997; 42: 223-241.

15 Whitehead WE, Palsson OS, Gangarosa L, Turner M, Tucker J. Lubiprostone does not influence visceral pain thresholds in patients with irritable bowel syndrome. Neurogastroenterol Motil 2011; 23: 944-e400.

16 Awad RA, Camacho S, Martin J, Rios N. Rectal sensation, pelvic floor function and symptom severity in Hispanic population with irritable bowel syndrome with constipation. Colorectal Dis 2006; 8: 488-493.

17 Houghton LA, Fell C, Whorwell PJ, Jones I, Sudworth DP, Gale JD. Effect of a secondgeneration alpha2delta ligand (pregabalin) on visceral sensation in hypersensitive patients with irritable bowel syndrome. Gut 2007; 56: 1218-1225.

18 Posserud I, Syrous A, Lindstrom L, Tack J, Abrahamsson H, Simren M. Altered rectal perception in irritable bowel syndrome is associated with symptom severity. Gastroenterology 2007; 133: 1113-1123.

19 Kanazawa M, Watanabe S, Tana C, Komuro H, Aoki M, Fukudo S. Effect of 5-HT4 receptor agonist mosapride citrate on rectosigmoid sensorimotor function in patients with irritable bowel syndrome. Neurogastroenterol Motil 2011; 23: 754-e332.

20 Awad RA. Altered recto-anal motility in irritable bowel syndrome: a clinical physiological study of 80 Mexican patients. J Gastrointest Motil (currently Neurogastroenterol Motil) 1993; 5: 265-271.
21 Fox M, Thumshirn M, Fruhauf H, Fried M, Schwizer W. Determinants of fecal continence in healthy, continent subjects: a comprehensive analysis by anal manometry, rectal barostat and a stool substitute retention test. Digestion 2011; 83: 46-53.

22 Bharucha AE, Edge J, Zinsmeister AR. Effect of nifedipine on anorectal sensorimotor functions in health and fecal incontinence. Am J Physiol Gastrointest Liver Physiol 2011; 301: G175-G180.

23 Peirce C, O'Herlihy C, O'Connell PR, Jones JF. Effect of injury on S1 dorsal root ganglia in an experimental model of neuropathic faecal incontinence. Br J Surg 2011; 98: 1155-1159.

24 Gladman MA, Aziz Q, Scott SM, Williams NS, Lunniss PJ. Rectal hyposensitivity: pathophysiological mechanisms. Neurogastroenterol Motil 2009; 21: 508-5.

25 Burgell RE, Bhan C, Lunniss PJ, Scott SM. Fecal incontinence in men: coexistent constipation and impact of rectal hyposensitivity. Dis Colon Rectum 2012; 55: 18-25.

26 Wijffels NA, Angelucci G, Ashrafi A, Jones OM, Cunningham C, Lindsey I. Rectal hyposensitivity is uncommon and unlikely to be the central cause of obstructed defecation in patients with high-grade internal rectal prolapse. Neurogastroenterol Motil 2011; 23: 151-154, e30.

27 Goodman SN. Confidence limits vs power, calculations. Epidemiology 1994; 5 266-268.

28 Loew BJ, Siegel CA. Foam preparations for the treatment of ulcerative colitis. Curr Drug Deliv 2012; 9: 338-344.

29 Kojima Y, Nakagawa T, Katsui R, Fujii H, Nakajima Y, Takaki M. A 5-HT4 agonist, mosapride, enhances intrinsic rectorectal and rectoanal reflexes after removal of extrinsic nerves in guinea pigs. Am J Physiol Gastrointest Liver Physiol 2005; 289: G351-G360. 